\title{
Particulate vaccine for bacterial diseases
}

\author{
Hariprasad O. ${ }^{*}$, Gopinath V.P. ${ }^{2}$, Navya A. ${ }^{3}$, Yugandhar V.G. ${ }^{1}$ and Sarma \\ P.V.G.K. ${ }^{1}$ \\ ${ }^{1}$ Department of Biotechnology, Sri Venkateswara Institute of Medical Sciences \& \\ SVIMS University, Tirupati, Andhra Pradesh, India. e-mail:hari.osuru@gmail.com. \\ ${ }^{2}$ Department of Animal Biotechnology, Madras Veterinary College, Chennai, India. \\ ${ }^{3}$ Department of Applied Microbiology, Sri Padmavati Mahila Visvavidyalayam, Tirupati, \\ Andhra Pradesh, India
}

\begin{abstract}
Particulate vaccines made a remarkable break through in the vaccines and drug delivery systems. With their fine small structure they can pass through the cellular systems and can trigger the type I and type II immune systems, which aid in the better immune response. The sero conversation and the longevity of the particulate vaccines are found to be better than the regular conventional vaccines. The development of recombinant vaccines and sub unit vaccines having safer side in vaccination could not elicit stronger immune response, because of their partial antigenicity. In such cases when coupled with nanoparticles triggers better immune response. Recombinant Tetanus toxoid and Diphtheria toxoid when coupled with nanoparticles showed significant TH1 and TH2 immune responses. Particulate vaccines can be used in mucosal, nasal, ocular or transcutaneous modes for vaccination.

Key words: Immune response, Sero conversation, Antigenicity, Nanoparticles and Vaccination.
\end{abstract}

\section{Introduction}

The advent of vaccines is one of the medicine's greatest findings. There is much research on particulate vaccines for viruses but less on bacteria. Our present review is focused on bacterial particulate vaccines. In recent years, significant effort was devoted to nanoparticle based drug delivery system, since it offers a suitable means of delivery system for delivering macromolecules like proteins, peptides, or genes to the target tissue site [1]. Because of their smaller size $(10-100 \mathrm{~nm})$ they can pass through the fine capillaries and can be easily taken up by the liver cells also [2]. Apart from targeted delivery, particulate vaccines have many other advantages like they prevent enzymatic degradation of some DNA vaccines, and type I immune response [3]. Through out the history most vaccines have been developed using live attenuated organisms, heat killed whole organism or inactivated toxins. Live vaccines can produce both humoral as well as cellular mediated immunity but, there is a chance for reverting back to the virulent form. Inactivated or heat killed are generally weak stimulators of immune response [4]. Recent efforts are focused on developing recombinant vaccines or subunit vaccines. This was a part of the whole antigen [5]. These antigens can't revert back to the wild form hence, they are highly safe. With the development of these types of vaccines there exists always a need for a delivery system. A simple system which can carry the antigen to the target site with out degrading them is the key role to be played by these nanoparticles [6]. When the solid nanoparticles are unstable physico-chemically they can be trapped inside the capsules and can be delivered without destroying their property [7]. Dendritic cells play a central role in the generation and attenuation of adaptive immune responses through interactions with other immune cells. During maturation, dendritic cells up regulate their chemokine receptors, as well as molecules essential for the activation of Tcells molecules and Co-stimulatory molecules. Final maturation of dendritic cells occurs in the lymph node and requires the interaction of $C D 40$ and $C D$ 40L [8]. Dendritic cells are increasingly well documented, comparatively little is known about its activation by microparticulate delivery system [9]. The uptake of microparticulate adjuvants by dendritic cells activates the NALP3 (Nacht Domain-, Leucine-Rich Repeat-, and PYDContaining Protein 3) inflammasome, and this contributes to their enhancing effects on innate and antigen-specific cellular immunity [10]. Anthrax cause by Bacillus anthracis, a Gram positive rod shaped bacteria. The key factor in anthrax's lethality is the ability to produce a tripartite 
toxin following spore germination. This lethal toxin consists of edema factor (EF), lethal factor (LF) and protective antigen (PA). It was known that PA neutralizing antibody is the main correlate of protection against anthrax. The recombinant protective antigen was prepared and coupled with poly -L-lactide (PLA) using a double emulsification, solvent evaporation method. The recombinant antigens when coupled with PLA nanoparticles showed higher activation of immune system when compared to uncoupled recombinant antigens alone [11]. Diphtheria vaccine was well incorporated in the nanocapsules made of chitosan nanoparticles by precipitation and coacervation method. These chitosan nanoparticles are positive particles with $10 \mathrm{~nm}$ size so they had a 100 percent loading efficiency and stability of more than 3 months. It showed good immune response [3]. The use of nanocarrier based mucosal vaccine delivery provides a suitable way for the nasal delivery of antigenic molecules. The nasal delivery compared to other mucosal sites is especially a finer way of immunization, as the nasal epithelium is characterized by relatively high permeability, low enzymatic activity and the presence of immunocompetent cells. In addition, the nasal route could offer simplified and more cost-effective protocols for vaccination with improved patient acquiescence. Moreover, the improved protection and facilitated transport of the antigen, nanoparticulate delivery systems could also provide more effective antigen recognition by immune cells. It represents the optimal processing and presenting of the antigen, further subsequent development of a suitable immune response [1]. Nanospheres are prepared by water -oil - water emulsion and solvent evaporation technique. Nearly all the reports describe the use of soluble forms of $\mathrm{CpG}$ Synthetic oligodeoxynucleotides (ODN) and antigens. The co-delivery of $\mathrm{CpG}$ ODN and antigens in biodegradable nanospheres is an alternative approach for immunization using tetanus toxoid (TT) as the model antigen and ODN \#1826 as the model CpG sequence. TT and CpG ODN were co-encapsulated in PLGA [poly (D,Llactic-co-glycolic acid) nanospheres found to have 73 percent coupling efficiency to tetanus toxoid. The co-delivery of $\mathrm{CpG}$ ODN adjuvants and antigens in nanospheres is a more efficient approach for immunization than the use of $\mathrm{CpG}$ ODN and TT in solution. These nanospheres showed very high sero conversion and elicited high immune response [12].

\section{Conclusion}

With the advent of naoparticles the immuogenicity of the antigen and the potency of the adjuvant were substantially increased. It's worth spending or depending further on the development of slow delivery vehicles. Using these nanoparticles the longevity of the immune response can be enhanced. It would be better to develop a good particulate system for delivering vaccines focused on bacterial diseases suitable for human and animals. However, very few trials were conducted with the nanoparticle coupled bacterial vaccines because they are not intra cellular. Thus, the design of optimized vaccine nanoparticles offers a propitious way for bacterial vaccination.

\section{References}

[1] Noemi Csaba, Marcos GarciaFuentes and Maria Jose Alonso. (2009) Advanced Drug Delivery Reviews, 61, 140-157.

[2] Jayanth Panyama and Vinod L. (2003) Advanced Drug Delivery Reviews, 55, 329347.

[3] Van der Lubben I.M., Verhoef J.C., Borchard G. and Junginger H.E. (2001) AdvancedDrug Delivery Reviews, 52,139-144.

[4] Laura J. Peek, Russell Middaugh C. and Cory Berkland (2008) Advanced DrugDelivery Reviews, 60, 915-928.

[5] Samantha Jilek, Hans $P$. Merkle and Elke Walter. (2005) Advanced Drug Delivery Reviews, 57, 377390.

[6] Omid C. Farokhzad and Robert Langer (2006) Advanced Drug Delivery Reviews, 58, 1456-1459.

[7] Yoshiaki Kawashima (2001) Advanced Drug Delivery Reviews, 47, 1-2.

[8] Tazio Storni; Thomas M. K., Gabriela Senti and Pal Johansen. (2005) Advanced 
DrugDelivery Reviews, 57, 333-355.

[9] Dang J.M. and Leong K. W. (2006) Advanced Drug Delivery Reviews, 58, 487499.

[10]Fiona A. S., Darren R., Benjamin C., Emma C., James H., Padma M., Manomohan S., Derek T. O'Hagan, Virginie P., Jurg T., Luke A. J. O'Neill. and Ed C.
Lavelle. (2009) Proceedings of the National Academy of Sciences of the United States of America, 106(3), 870-875.

[11]Angie westwood, Gareth D. Healey, Diane williamson E. and Jim Eyles E. (2005) Vaccine, 23, 3857-3863.

[12]Manish Diwan; Mohsen Tafaghodi and John Samuel. (2002) Journal of Controlled Release, 85, 247-262. 